\title{
EEF1D Promotes Glioma Proliferation, Migration, and Invasion through EMT and PI3K/Akt Pathway
}

\author{
Cheng Xie $\mathbb{D}^{1},{ }^{1}$ Mingfeng Zhou, ${ }^{1}$ Jie Lin, ${ }^{1}$ Zhiyong Wu, ${ }^{2}$ Shengfeng Ding, ${ }^{1}$ Jie Luo, ${ }^{1}$ \\ Zhengming Zhan, ${ }^{1}$ Yonghua Cai, ${ }^{1}$ Shuaishuai Xue, ${ }^{1}$ and Ye Song ${ }^{1}{ }^{1}$ \\ ${ }^{1}$ Department of Neurosurgery, Nanfang Hospital, Southern Medical University, Guangzhou, 510515 Guangdong, China \\ ${ }^{2}$ Department of Neurosurgery, The Second Affiliated Hospital of the Chinese University of Hong Kong (Shenzhen), Shenzhen, \\ 518116 Guangdong, China
}

Correspondence should be addressed to Ye Song; songye@smu.edu.cn

Received 27 June 2020; Revised 12 August 2020; Accepted 21 August 2020; Published 25 September 2020

Academic Editor: Bing Niu

Copyright (c) 2020 Cheng Xie et al. This is an open access article distributed under the Creative Commons Attribution License, which permits unrestricted use, distribution, and reproduction in any medium, provided the original work is properly cited.

\begin{abstract}
Eukaryotic translation elongation factor $1 \delta$ (EEF1D), a subunit of the elongation factor 1 complex of proteins, mediates the elongation process of protein synthesis. Besides this canonical role, EEF1D was found overexpressed in many tumors, like hepatocarcinomas and medulloblastomas. In the present study, we demonstrated for the first time that EEF1D may interact with other putative proteins to regulate cell proliferation, migration, and invasion through PI3K/Akt and EMT pathways in glioma. Furthermore, knockdown of EEF1D could reduce cell proliferation and impaired epithelial-mesenchymal transition (EMT) phenotypes, including cell invasion. Taken together, these results indicate that EEF1D and its partner proteins might play a critical role in glioma and serve as a potential therapeutic target of glioma.
\end{abstract}

\section{Introduction}

Glioma, the most common and malignant primary brain tumor, is characterized by high-grade proliferation, invasion, and poor prognosis. Being an aggressive tumor, glioma also shows poor response to common therapeutic treatments including surgery, radiation, and conventional concomitant and adjuvant chemotherapy with temozolomide (TMZ). Although treatment methods advanced during the past decades, the median survival of glioma is still about 14.6 months and five-year survival is less than $10 \%[1-4]$. Considering that the molecular mechanism underlying glioma is still ambiguous, it is an urgent need to unravel mechanisms of proliferation, invasion, and resistance to identify weaknesses that may be exploited using new and existing agents to increase survival from glioma.

EEF1D, as a part of the eukaryotic translation elongation factor 1 (EEF1) complex, serves as the enzymatic delivery of aminoacyl tRNAs to the ribosome and functions as a guanine nucleotide exchange factor. Based on the canonical and noncanonical functions $[5,6]$, emerging amounts of evidence indicate that EEF1 proteins, particularly the prototypical member EEF1D, may play a role in the control of cellular processes during tumorigenesis [6]. In 2000, Shuda et al. reported a higher level of EEF1D mRNA in hepatocarcinomas [7], and in 2008, Piltti et al. reported the correlation of EEF1D with phosphorylation of ERK of the MAP kinase pathway in chondrosarcoma [8]. Later, with advances in detection approaches, such as chromosomal comparative genomic hybridization, two-dimensional electrophoresis coupled with MALDI-TOF-MS and quantitative PCR, the upregulation of EEF1D was described by several researchers in other cancers like esophageal carcinomas [9], non-smallcell lung cancers [10], and medulloblastomas [11]. However, little has been uncovered regarding the underlying biological mechanisms correlated with EEF1D overexpression in glioma, and the involvement of EEF1D in glioma tumorigenesis has not yet been investigated.

In the present study, we found that EEF1D may play a role in the development of glioma through multiple pathways and provided new insights into the glioma initiation. We also speculated that EEF1D may be involved in multiple major 
cell signaling pathways simultaneously like EMT and PI3K/ Akt pathways to regulate cell survival, migration, and invasion in glioma. Furthermore, EEF1D may constitute a new prospect for a therapeutic target against human glioma.

\section{Materials and Methods}

2.1. Bioinformatics Analysis. The genomic expression data and clinicopathological data of 163 GBM tissues, 518 LGG tissues, and 207 normal brain tissues were downloaded from The Cancer Genome Atlas (TCGA, https://tcga-data.nci.nih .gov/TCGA). The putative EEF1D-interacting proteins were subjected to functional annotation analysis using FunRich analysis tool $[12,13]$.

2.2. Cell Culture. The U87 and A172 glioblastoma cell lines were purchased from the American Type Culture Collection (ATCC, USA). All cell lines were cultured in Dulbecco's modified Eagle's medium (DMEM) (Hyclone, Logan, USA) supplemented with $10 \%$ fetal bovine serum (FBS, Hyclone, USA) and incubated in a humidified atmosphere under 5\% $\mathrm{CO}_{2}$ at $37^{\circ} \mathrm{C}$.

2.3. Collection of Clinical Samples. Clinical sample tissues were collected from patients afflicted with glioma. All samples were then confirmed pathological diagnosis and classified according to the World Health Organization (WHO) criteria. Every human tissue was obtained with advanced written informed consent from patients or their guardians before participation in the study, and approval from the Ethics Committees of Nanfang Hospital of Guangdong Province was obtained.

2.4. Western Blot. Western blot was performed as previously described. The cells or clinical tissues were washed thoroughly with PBS three times and lysed with RIPA Buffer (50 mM Tris- $\mathrm{HCl}$ pH 8.0, 1 mM EDTA pH 8.0, 5 mM DTT, $2 \%$ SDS) with a protease inhibitor and phosphoric-acid protease inhibitor under $4^{\circ} \mathrm{C}$ for 30 minutes. The protein concentration was then determined using BCA assay (Beyotime Inc., China). The proteins were separated subsequently using SDS-PAGE gel and electro-transferred to polyvinylidene difluoride membranes (Invitrogen, Carlsbad, CA). The membranes were blocked with 5\% BSA and then incubated with primary antibodies, including EEF1D (PTG, USA); E-cadherin, $\mathrm{N}$-cadherin, $\beta$-catenin, slug, snail, and vimentin (Cell Signaling Technology, USA); PI3K and PI3K (phosophoTyr467/199); Akt and p-Akt (phosphor-Ser473) (SAB, USA); and GAPDH (CWbio, China), overnight at $4^{\circ} \mathrm{C}$ and then incubated with horseradish peroxidase-conjugated secondary antibody for $1 \mathrm{~h}$ under room temperature. Finally, signals were detected using enhanced chemiluminescence reagents (Pierce, Rockford, IL, USA). All experiments were independently performed in triplicate.

2.5. IHC. For IHC, tissue sections underwent gradient ethanol dehydration and infiltration of histological samples in tissue processor, were embedded in paraffin and $4 \mu \mathrm{m}$ thin sectioned, and were then deparaffinized in xylene. After washing thoroughly with PBS, the slides were treated with
$10 \mathrm{mM}$ citrate buffer for 2 minutes at $100^{\circ} \mathrm{C}$ to achieve antigen retrieval and treated with $3 \%$ hydrogen peroxide to block endogenous peroxidase activity. After washing thoroughly with PBS, slides were blocked with 5\% BSA for 1 hour at room temperature and incubated with EEF1D primary antibody (PTG, USA) at $4^{\circ} \mathrm{C}$ overnight. After washing thoroughly with $\mathrm{PBS}$, the slides were incubated with biotin-labeled goat anti-mouse or anti-rabbit antibodies (ZSGBbio, China) for 1 hour at room temperature. The sections were finally visualized with $\mathrm{DAB}$, counterstained with hematoxylin, mounted in neutral gum, and imaged using a bright field microscope equipped with a digital camera (Leica, Germany).

2.6. Small Interfering RNA (siRNA) Transfection. Transfection of siRNA was performed according as previously described [14]. For EEF1D knockdown, siRNA targeting EEF1D along with the negative control was designed and synthesized by RiboBio (Guangzhou, China). The sequences to EEF1D were GCCGCAACAUCUUAGGGAA (siEEF1D-1), GCAACAUCUUAGGGAACAA (siEEF1D-2), and CCUUGC CCUACUGUUACUU (siEEF1D-3). Among three siRNAs targeting on EEF1D gene, the most effective one (siEEF1D) was identified by Western blot and applied for the further experiments. Glioma cells U87 and A172 were plated onto a 6-well plate at 30-50\% confluence. After incubation for 6 hours at $37^{\circ} \mathrm{C}$ in a $5 \% \mathrm{CO}_{2}$ atmosphere, siEEF1D mixed with Lipo2000 (Thermo Fisher, USA) was then transfected into cells following the manufacturer's protocol. Cells were then collected after 24-48 hours for further experiments.

2.7. Transwell and Boyden Assays. In vitro cell migration Transwell assay and invasion Boyden assay were performed according as previously described [14]. For the Transwell assay, $1 \times 10^{4}$ cells in $100 \mu \mathrm{l}$ DMEM medium without FBS were seeded onto the upper chamber of the Transwell apparatus (Costar, MA), and $500 \mu \mathrm{l}$ DMEM with $10 \%$ FBS was added into the lower chamber as a chemoattractant. After incubation for 6 hours at $37^{\circ} \mathrm{C}$ in a $5 \% \mathrm{CO}_{2}$ atmosphere, the fibronectin-coated polycarbonate membrane insert was washed with PBS, and cells adhering to the top surface of the insert were removed with a cotton swab. Cells on the lower surface were then fixed with methanol, stained with crystal violet solution, and counted under a microscope in five predetermined fields $(200 \mathrm{x})$. All assays were independently repeated at least thrice. For the Boyden assay, the procedure was similar to the above one, except for the fact that the Transwell membranes were first precoated with $24 \mu \mathrm{g} / \mu \mathrm{l}$ Matrigel (R\&D Systems, USA), and the cells were incubated in the Transwell apparatus for 8 hours at $37^{\circ} \mathrm{C}$ in a $5 \% \mathrm{CO}_{2}$ atmosphere. Cells on the lower surface were counted in the same way as the cell migration assay.

2.8. Immunoprecipitation. For immunoprecipitation, glioma U87 cells were rinsed once with cold PBS and lysed with Pierce $^{\mathrm{TM}}$ IP Lysis Buffer (Thermo Fisher, USA). Lysates were kept on ice for 10 minutes and centrifuged at $12,000 \mathrm{rpm}$ for 15 minutes. The supernatant was then incubated for 4 hours with protein A/G beads (Bimake, China) preloaded 


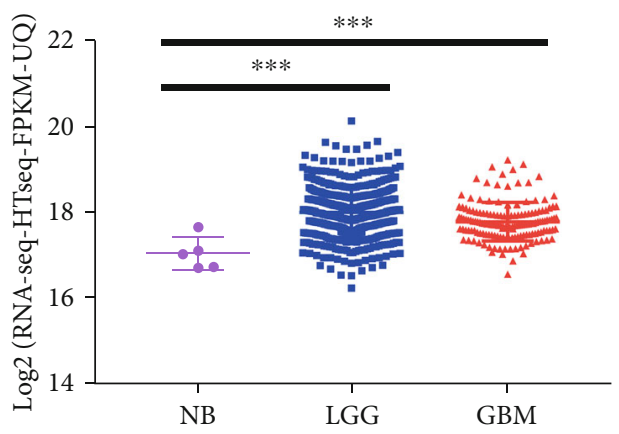

(a)

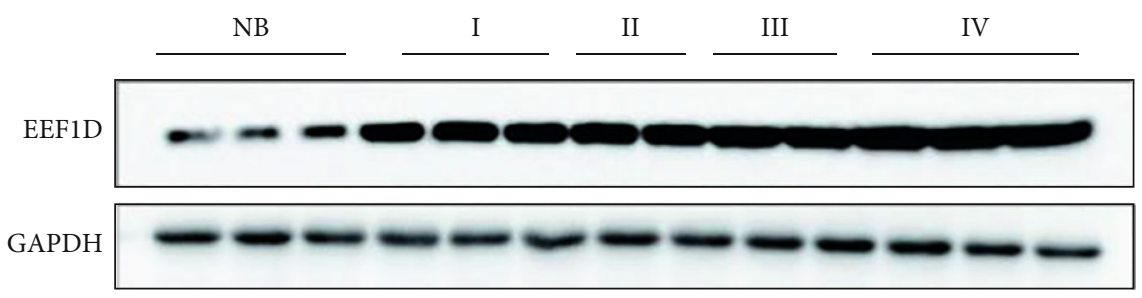

(b)

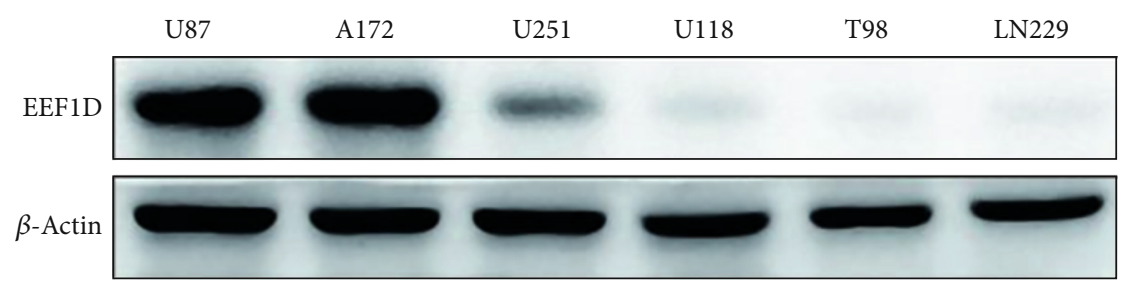

(c)

FIGURE 1: Expression of EEF1D in LGG, GBM, and nontumor brain tissues. (a) EEF1D expressions at mRNA level in normal brain tissues $(N=5)$, low-level glioma (LGG) $(N=511)$, and glioblastoma $(\mathrm{GBM})(N=156)$ were analyzed utilizing TCGA database. (b). EEF1D expressions at protein level were analyzed in clinical samples, including nontumor brain tissues $(N=3)$, grade I $(N=3)$, grade II $(N=2)$, grade III $(N=2)$, and grade IV $(N=3)$. (c). EEF1D expressions at protein level were analyzed in six glioma cell lines (U87, A172, U251, U118, T98, and LN229).

with EEF1D antibody (PTG, USA) at room temperature. The immunoprecipitate was rinsed three times with washing buffer (Thermo Fisher, USA), suspended in SDS loading buffer and boiled for 8 minutes at $95^{\circ} \mathrm{C}$. Immunoprecipitated proteins were analyzed by SDS-PAGE/Western blotting.

2.9. Liquid Chromatograph-Mass Spectrometer (LC-MS) Analysis. In brief, immunoprecipitated peptides were applied for further liquid chromatograph-mass spectrometry (LCMS) analysis using a micro HPLC (high pressure liquid chromatography) system connected to an LCQ Deca XP-plus ESI ion-trap mass spectrometer. Chromatographic separation is performed by normal-phase HPLC on a TSK-gel Amide-80 $(3 \mu \mathrm{m})$ column $(2 \times 150 \mathrm{~mm})$. The LC-MS parameters were determined as described previously [15], and the mass spectra were obtained by ion monitoring based on $\mathrm{m} / \mathrm{z}$.

\section{Results}

3.1. Expression of EEF1D in LGG, GBM, and Nontumor Brain Tissues. By analyzing the data from TCGA database, we characterized EEF1D expression in 5 cases of nontumor brain tissues, 511 cases of LGG samples, and 156 cases of
GBM samples. EEF1D expression was significantly higher in LGG and GBM samples $(P<0.01)$ (Figure $1(\mathrm{a}))$. Besides, consistent with TCGA results, EEF1D was increased in LGG and GBM compared to normal brain tissues by immunoblotting (Figure 1(b)). Consistently, we further confirmed higher expression in high-grade glioma than low-grade glioma using IHC staining and the IHC score (Figure 2). Moreover, we also detected higher EEF1D expression in U87 and A172 glioblastoma cell lines among six commonly used glioma cell lines (Figure 1(c)), so U87 and A172 glioblastoma cell lines were chosen for a further experiment.

3.2. Inhibition of EEF1D Impaired Proliferation, Migration, and Invasion of Glioma Cells. To investigate the influence of EEF1D on glioma cells, we constructed siRNA targeting EEF1D gene. As expected, EEF1D expression was significantly decreased in both U87 and A172 cells after transfection with EEF1D-siRNA (Figures 3(a) and 3(b)), which in turn resulted in significant inhibition of glioma cell proliferation (Figures 3(c) and 3(d)). Transwell assay further determined that depletion of EEF1D could impair the migration capacity of U87 and A172 cells (Figures 3(e) and 3(f)) and resulted in lower invasion capacity compared to negative 

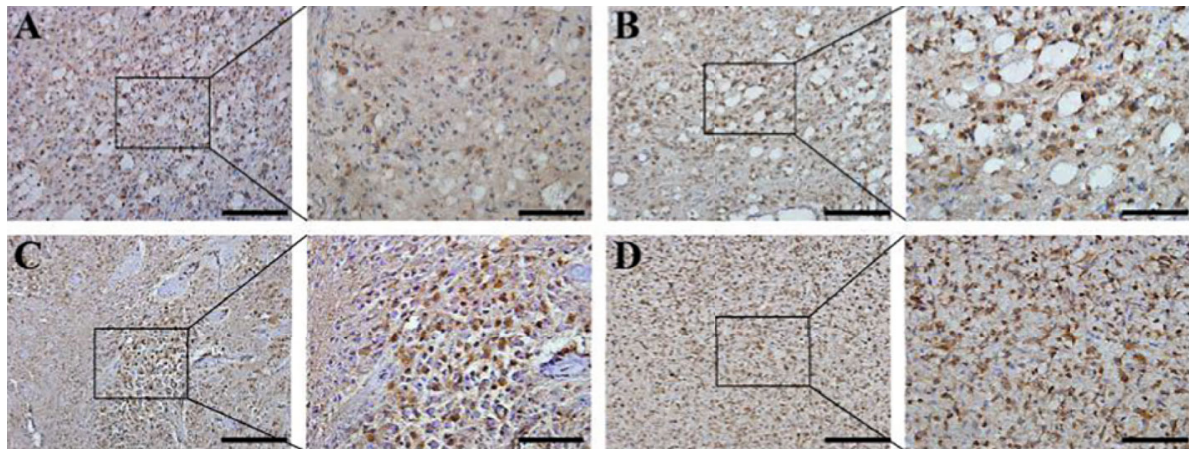

(a)

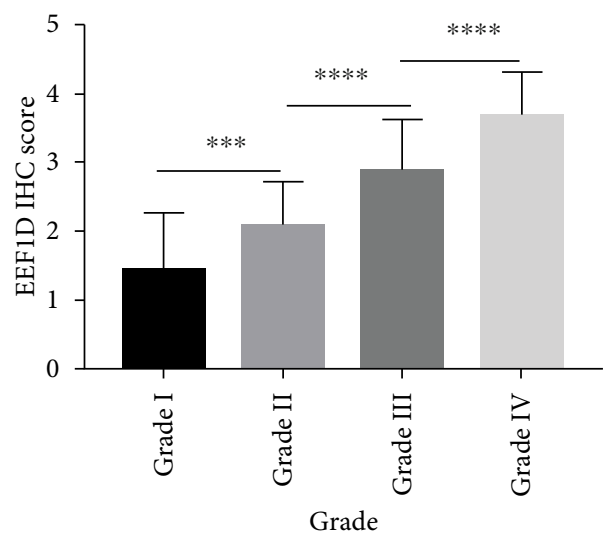

(b)

FIgURE 2: Expression of EEF1D in different grade glioma tissues by immunohistology. (a) Representative immunohistochemical staining samples of different grade human glioma (A - grade I, B-grade II, C-grade III, and D—grade IV) with anti-EEF1D antibody. Scale bars: $200 \mu \mathrm{m}$ for low magnification image and $100 \mu \mathrm{m}$ for high magnification image. (b) The IHC score of different clinical sample tissues (including grade I $(n=14)$, grade II $(n=38)$, grade III $(n=31)$, and grade IV $(n=25))$ was analysed by using an independent samples $t$ test $(* * * P<0.001, P=0.0043 ; * * * * P<0.0001)$.

control group, as Boyden assays have shown (Figures 3(g) and $3(\mathrm{~h})$ ). In summary, these results proposed that inhibition of EEF1D could suppress the cell growth and reduce the malignant phenotype-like proliferation, migratory, and invasion of glioma cells.

3.3. Knockdown of EEF1D Could Regulate the Expression of EMT Markers and Suppressed the Activation of PI3K/Akt Signaling Pathway. To investigate the underlying mechanism of EEF1D, the protein levels of EMT markers and PI3K/Akt signaling pathway were measured to further determine the effects of EEF1D suppression after transfection with EEF1D-siRNA in U87 and A172 glioma cells. As shown in Figures 4(a) and 4(b), levels of the epithelial marker Ecadherin were slightly promoted, whereas the expression of mesenchymal markers including $\mathrm{N}$-cadherin and Snail was significantly downregulated in U87 and A172 glioma cells following EEF1D-siRNA transfection. Further, $\beta$-catenin, a critical transcriptional factor of the EMT process, was also decreased after EEF1D knockdown. Taken together, these findings suggested that EEF1D was closely associated with the modulation of EMT progress in glioma cells, and inhibition of EEF1D could reverse the EMT characteristics of glioma cells.
The PI3K/Akt pathway has been identified as a key regulator to growth and proliferation of malignant glioma cells. In the present study, Western blot results revealed that PI3K, p-PI3K, Akt, and p-Akt in glioma cells transfected with EEF1D-siRNA were significantly lower than those transfected with NC-siRNA $(P<0.05$; Figure $4(\mathrm{c}))$. Thereby, the downregulation of EEF1D expression suppressed the activation of the PI3K/Akt pathway, which in turn might impede cell proliferation.

3.4. Identification of EEF1D Interacting Proteins. In order to further figure out the regulatory network of EEF1D on several signaling pathways, we carried out immunoprecipitation assays combined with LC-MS/MS to achieve qualitative and quantitative analyses of the potential EEF1D-interacting partners. As expected, EEF1D was immunoprecipitated with the EEF1D-specific antibody while no protein amounts were detected in the control IgG sample (Figure 5(a)). The top 20 putative specific interactors are listed in Table 1. Next, GO functional and KEGG pathway enrichment analyses were performed using the software of enrichment analysis tool FunRich: Functional Enrichment analysis tool [16-18]. Based on their primary biological processes, the majority of these proteins were considered involved in distinct functions. 


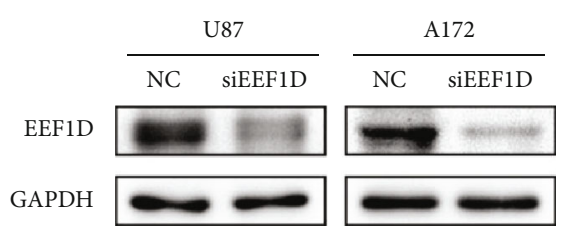

(a)

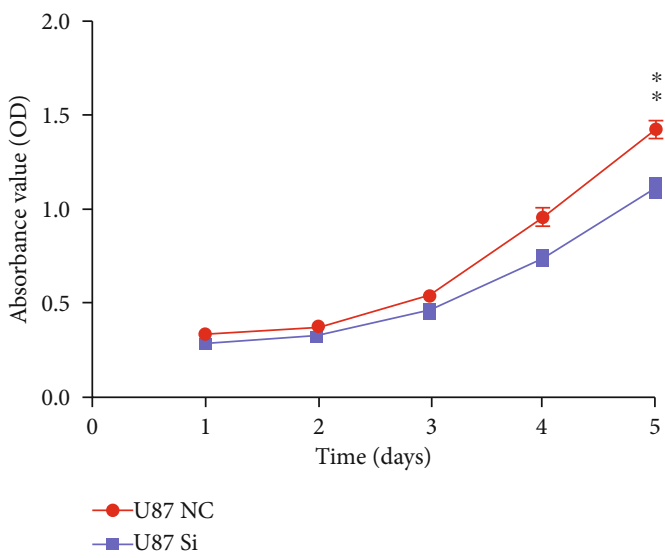

(c)

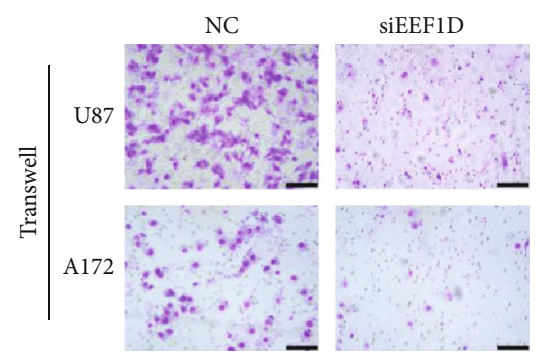

(e)

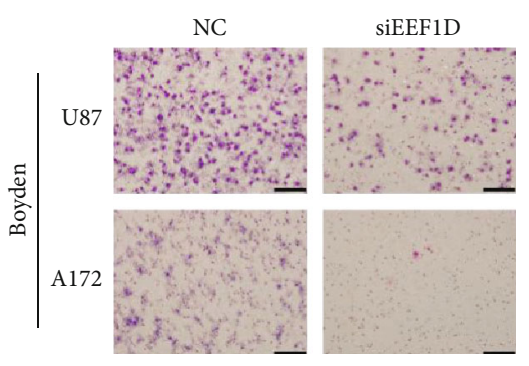

$(\mathrm{g})$
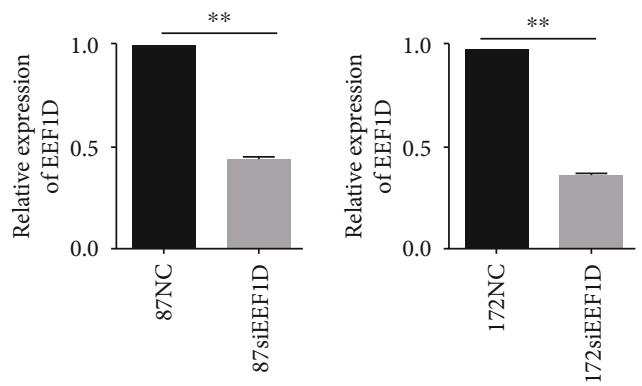

(b)

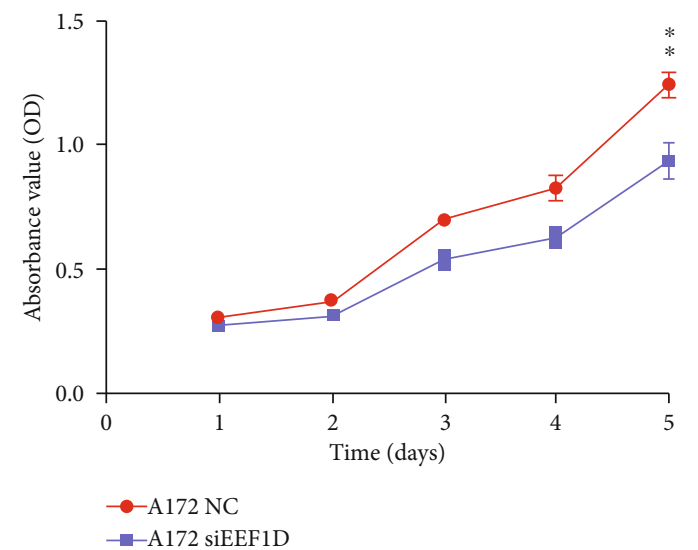

(d)
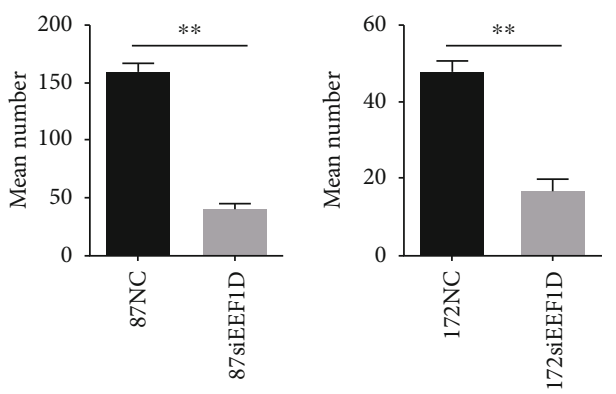

(f)
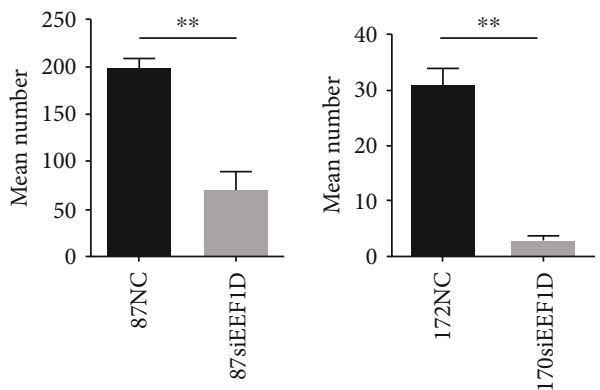

(h)

FIGURE 3: Inhibition of EEF1D impaired proliferation, migration, and invasion of glioma cells. (a) Efficacy of EEF1D-siRNA on EEF1D expression in U87 and A172 glioma cells, respectively. (b) Quantification results of (a). (c) Effect of EEF1D on proliferation of U87 and A172 glioma cells in vitro. The U87 and A172 glioma cells were subjected to CCK- 8 assay for 5 d. There was a significant difference between two groups at day 5. NC: negative control siRNA-transfected cells; siEEF1D: EEF1D-siRNA transfected cells $\left({ }^{* *} P<0.01\right.$ as compared with NC). (d) Quantification results of (c). (e, g) Transwell assay and Boyden assay were performed to determine cell migration and invasion in glioma U87 and A172 cell transfected with EEF1D-siRNA or negative control siRNA, respectively. NC: negative control siRNA-transfected cells; siEEF1D: EEF1D-siRNA-transfected cells $\left({ }^{* *} P<0.01\right.$ as compared with NC). Scale bars: $100 \mu$ m. (f, h) Quantification results of (c) and (e) respectively. 

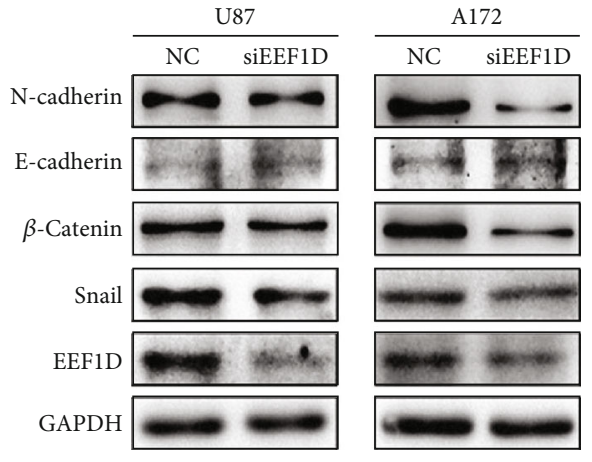

(a)
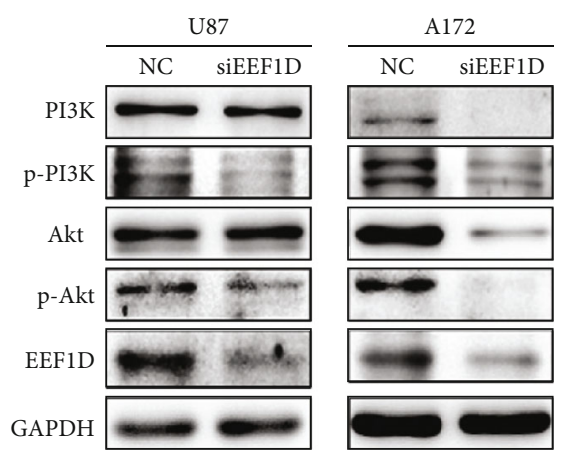

(c)
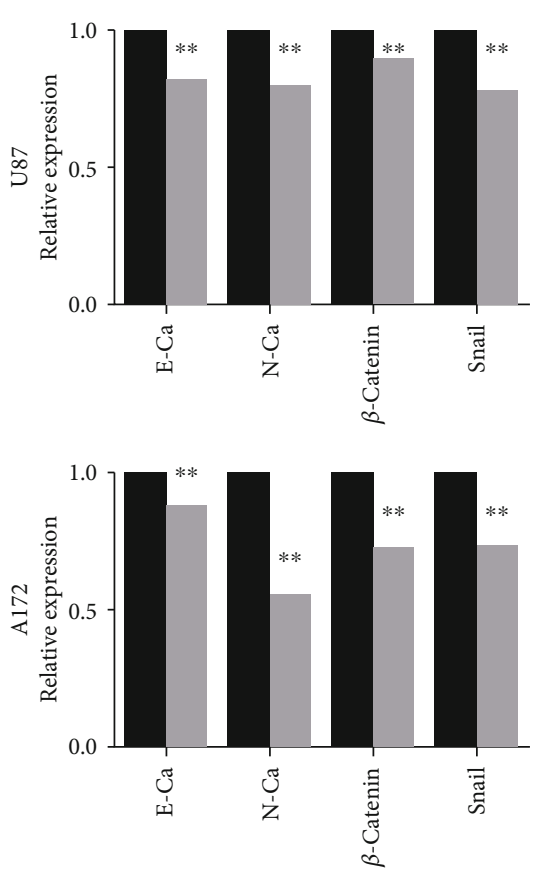

NC

siEEF1D

(b)
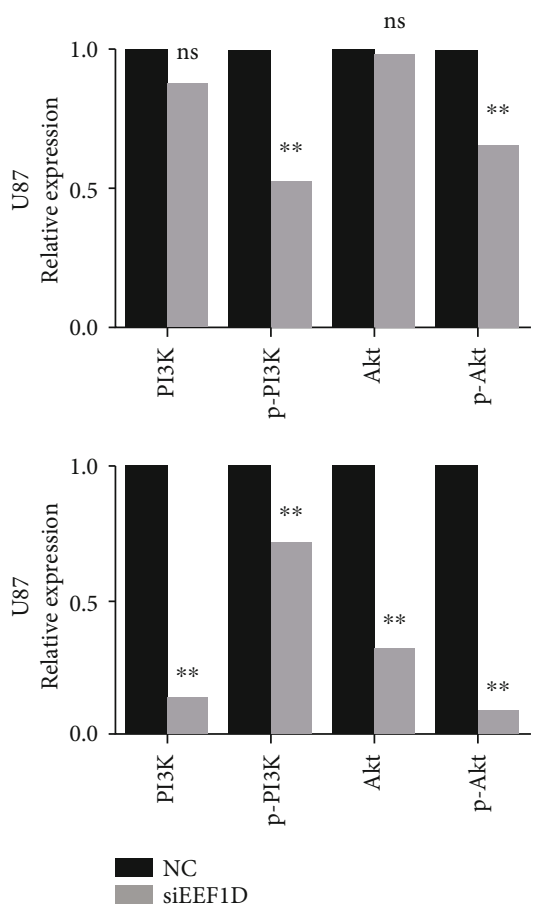

(d)

FIGURE 4: Downregulation of EEF1D could regulate the expression of EMT markers and suppressed the activation of the PI3K/AKT signaling pathway. (a) Protein levels of EMT markers were detected in U87 and A172 glioma cells by Western blot. GAPDH was also detected as the control of sample loading. NC: negative control siRNA-transfected cells. siEEF1D: EEF1D-siRNA-transfected cells. Data were based on at least three independent experiments and shown as the mean $\pm \mathrm{SD}\left({ }^{* *} P<0.01\right.$ as compared with NC). (b) Quantification results of (a). (c) Protein levels of PI3K/Akt signal pathway were detected in U87 and A172 glioma cells by Western blot. GAPDH was also detected as the control of sample loading. NC: negative control siRNA-transfected cells; siEEF1D: EEF1D-siRNA-transfected cells. Data were based on at least three independent experiments and shown as the mean $\pm \mathrm{SD}\left({ }^{* *} P<0.01\right.$ as compared with NC). (d) Quantification results of (c). 


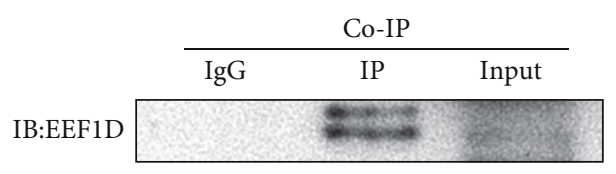

(a)

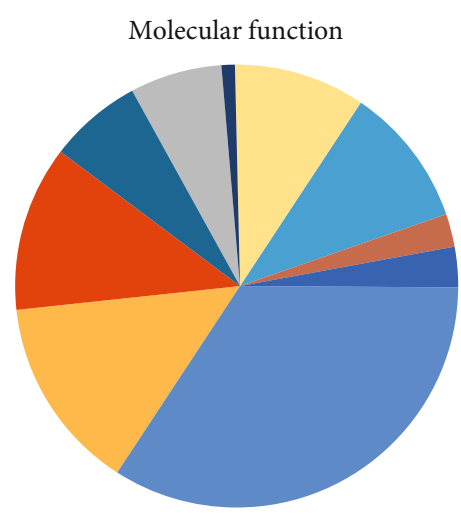

RNA binding

Ribosome structure

Cytoskeleton structure

Ribonucleoprotein

Translation regulating

Nucleocytoplasmic transport

Cytoskeleton protein binding

Structure molecule activity

Transaminase activity

Cytoskeletal anchoring

(b)

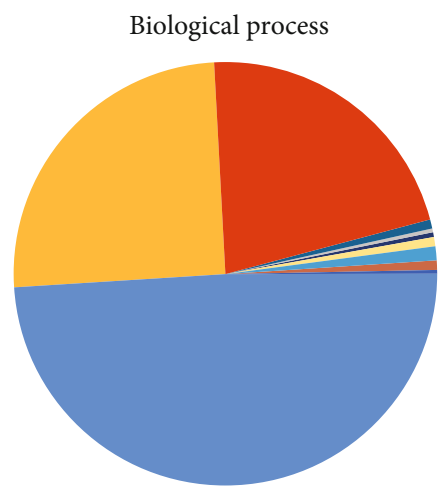

Nucleic acid metabolism

Cell growth

Protein metabolism

Mitosis

Nuclear biogenesis

ER organization

Protein transport

RNA metabolism

Translation regulation

Spindle assembly

(d)

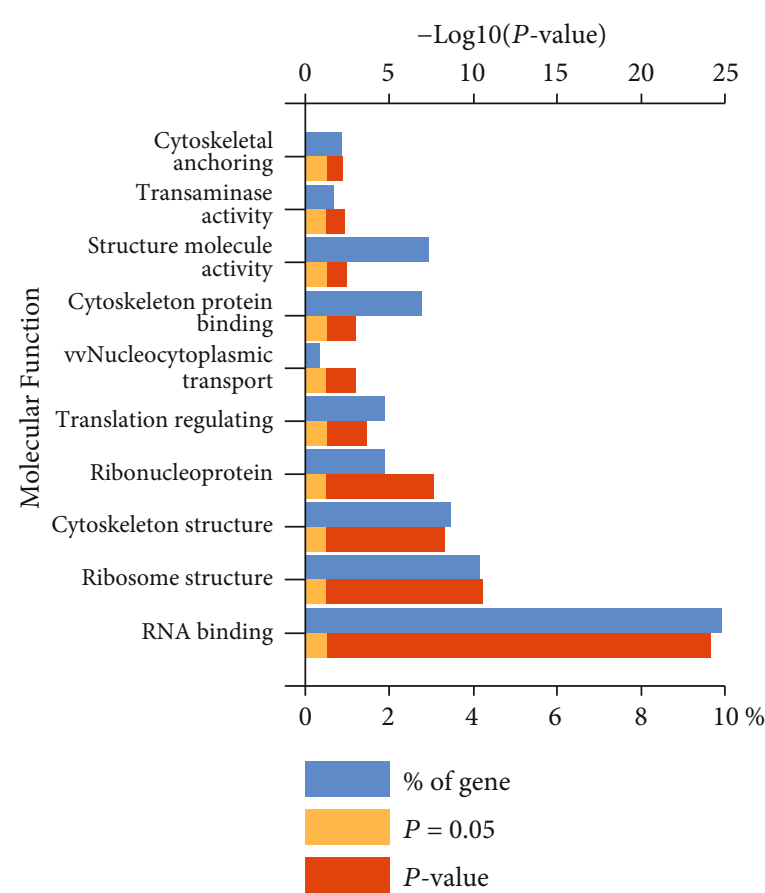

(c)

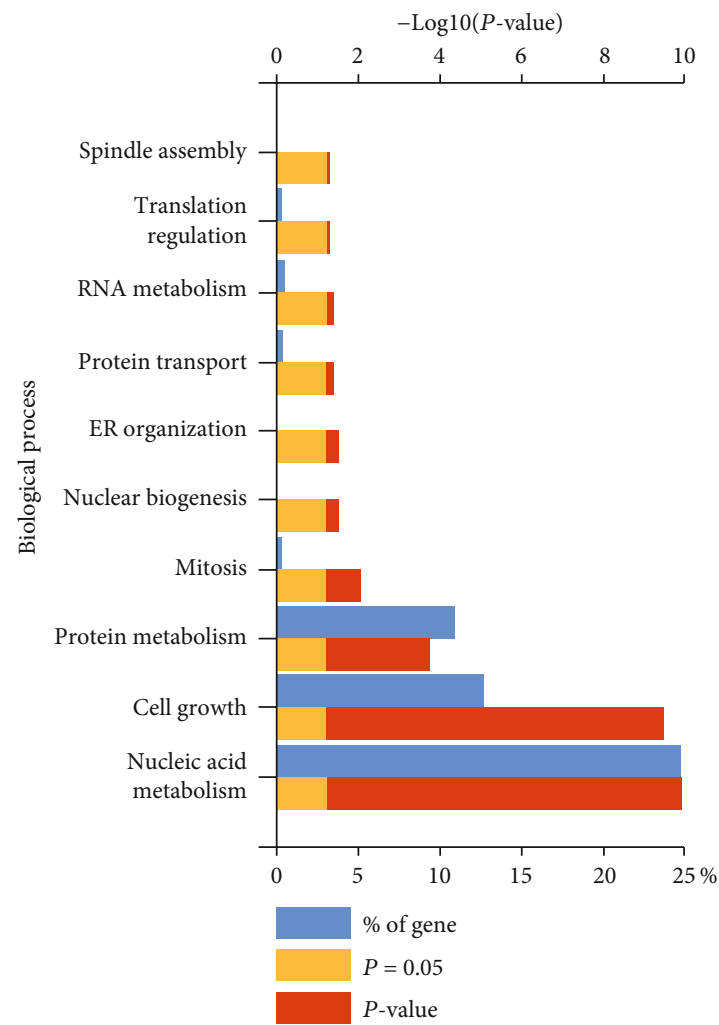

(e)

Figure 5: Continued. 


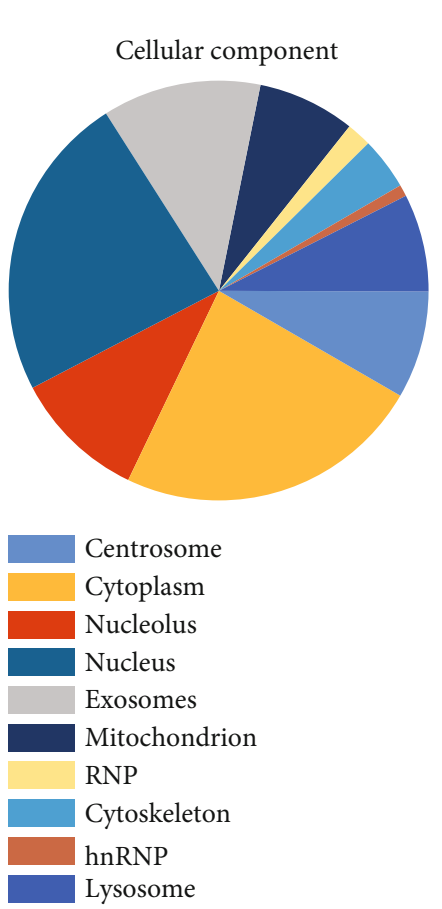

(f)

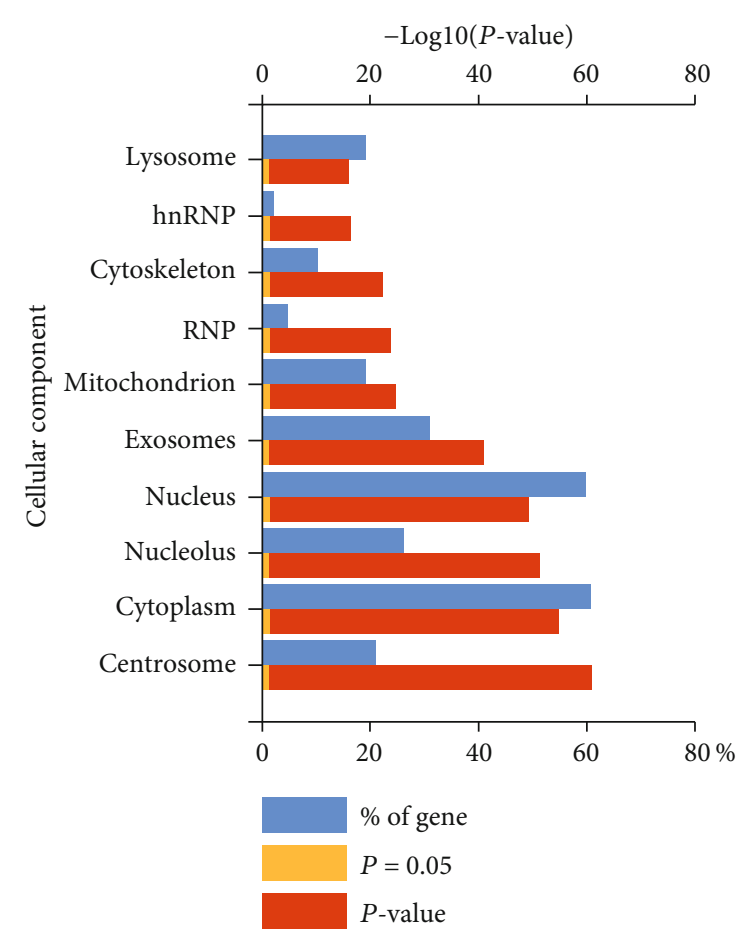

(g)

FIGURE 5: Identification of EEF1D interacting proteins. (a) Immunoprecipitation assays showing a good efficacy of specific EEF1D antibody. The top 10 enriched ( $b$ and $c$ ) molecular function (Venkatesan, Lamfers et al.) pathways, ( $\mathrm{d}$ and e) biological process (BP) pathways, and ( $\mathrm{f}$ and $\mathrm{g}$ ) cellular component (CC) pathways of putative EEF1D-interacting protein from GO analysis by FunRich.

The detailed enriched GO functions for the interacting proteins are presented in Supplement Excel. In aspect of molecular function (Venkatesan, Lamfers et al.), top 10 associated functions were RNA binding, structural constituent of ribosome, structural constituent of cytoskeleton, ribonucleoprotein, translation regulator activity, et al., as displayed in Figures 5(b) and 5(c). Thus, the annotation of the biological process (BP) showed that proteins immunoprecipitated with EEF1D were mainly related to the regulation of nucleobase, nucleoside, nucleotide, and nucleic acid metabolism; signal transduction; cell growth and/or maintenance; and protein metabolism (Figures 5(d) and 5(e)). In addition, distribution of interacting proteins according to cellular component (CC) was also noted (Figures 5(f) and 5(g)). Via the biological pathway analysis, we discovered that the nucleic acid metabolism and cell growth were the most significantly enriched. Therefore, we speculated that EEF1D was likely to be involved in DNA replication and RNA synthesis through binding catalytic proteins and regulating its capacity.

Taken together, these results indicated that EEF1D might bind to other proteins, like the protein of the EMT and PI3K/Akt pathway, to form a functional complex to regulate the progression phenotype of glioma cells.

\section{Discussion}

In the present study, aberrant expression of EEF1D was confirmed in glioma samples in comparison with nontumor brain tissues. Besides, blocking the EEF1D restrained the critical EMT and PI3K/Akt pathway. Furthermore, GO func- tional and KEGG pathway enrichment analyses revealed that EEF1D might bind to other proteins to mediate nucleic acid metabolism and cell growth. These results indicate that EEF1D may play a critical role in glioma cell proliferation, migration, and invasion and acts as an oncogene in glioma.

Proteomic analysis shows that EEF1D is overexpressed in right-sided colon cancer [19] and correlates with the invasive status of Adriamycin-resistant variants of DLKP, a squamous lung and cancer cell line [20]. Genomic analysis in medulloblastoma also reveals that a higher EEF1D level was adversely associated with outcome [11]. Isadora et al. reported that overexpressed EEF1D leads to the modulation of proliferation via cyclin D1 and EMT and invasion in oral squamous cell carcinoma [6]. Consistently, in the present study, we found that the expression of EEF1D, as indicated both by immunohistology staining and immunoblotting, was elevated and positively correlated with glioma grade.

Besides the canonical role of EEF1D as a direct substrate for casein kinase 2 (CK2), an important regulator of cell cycle progression, apoptosis, and transcription [21, 22], EEF1D, could also participate in autoubiquitination and degradation by interacting with SIAH E3 ubiquitin protein ligase 1 (SIAH-1) [23]. Several researchers reported that gene amplification, genetic methylation, and posttranslational modification may play a role in the mechanisms of EEF1 superfamily proteins in different cancers [24].

Epithelial-mesenchymal transition (EMT), represented by remarkable morphological changes from an epithelial cobblestone phenotype to an elongated fibroblastic phenotype, provides a new insight into the potential mechanism 


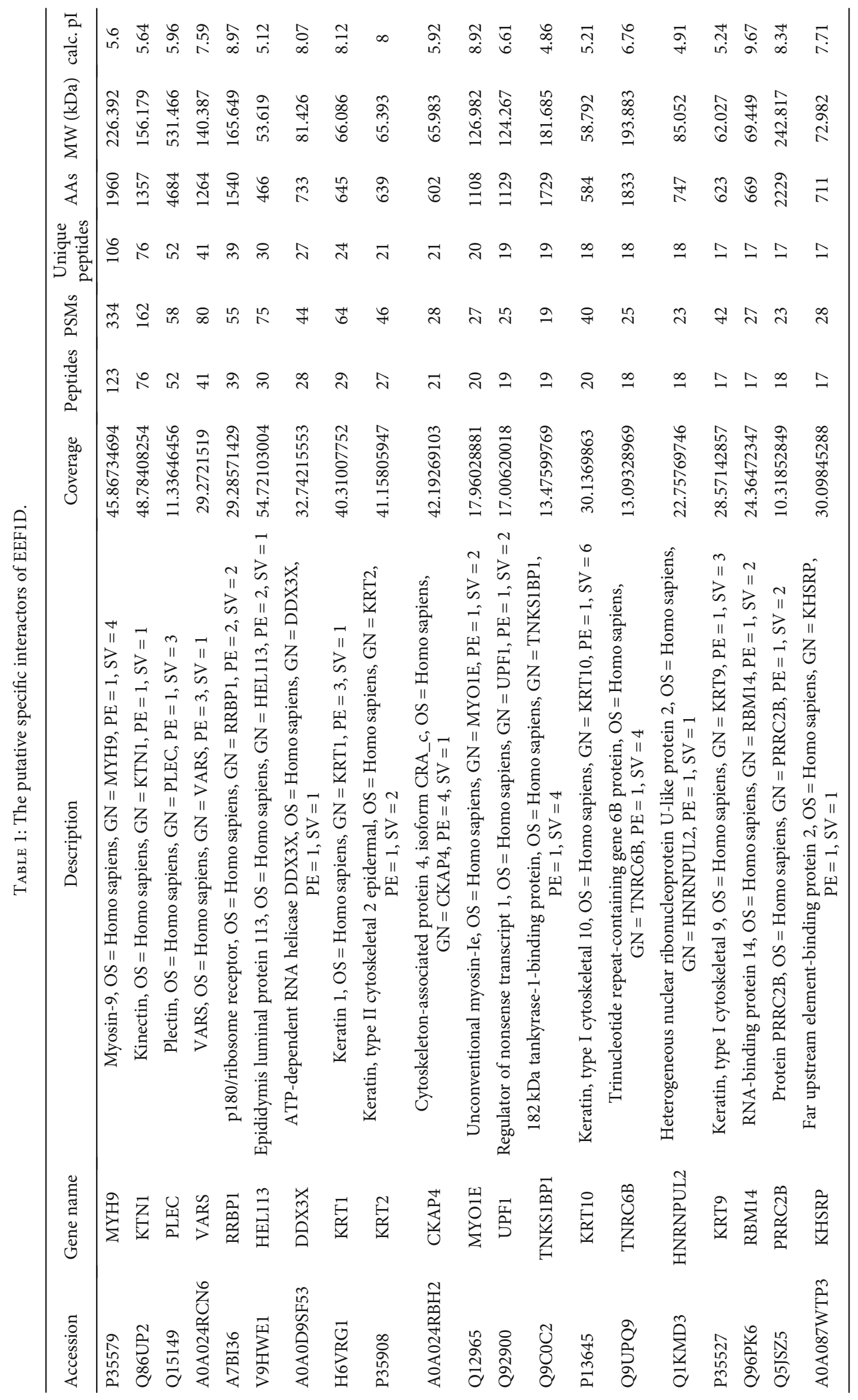


for the glioma metastasis and invasiveness. EMT is characterized by a decreased expression of epithelial markers, such as E-cadherin, and an increased expression of mesenchymal markers, such as $\mathrm{N}$-cadherin and vimentin, and transcription factors, such as slug, snail, and $\beta$-catenin [25-28]. EMT is a transcriptional process in which epithelial cells adopt mesenchymal properties by loss of cell-cell adhesion, acquisition of migratory and invasive properties, and loss of cell polarity, related to tumor initiation, invasion, metastasis to distant sites, and resistance to chemo- and/or radiotherapy $[27,28]$. The PI3K/Akt signaling pathway, a well-known pathway in the regulation of tumorigenesis, tumorigenesis, is also activated in glioma $[29,30]$. As an important intracellular signaling pathway, PI3K/Akt is known to be closely associated with the proliferation, migration, and progression of tumors, including glioma [31-33]. Besides, EMT pathways could cross talk with the PI3K/Akt/GSK $3 \beta$ pathway in glioma, through HIF- $1 \alpha$, PTEN, and the $\mathrm{WNT} / \beta$-catenin pathways [28].

Our results suggested that EEF1D exerts its effect on glioma by promoting EMT and PI3K/Akt signaling pathways, which could be the potential mechanism by which EEF1D promotes tumor progression.

According to the results of GO analysis of the EEF1D interacting protein, the most enriched molecular functions were involved in the regulation of RNA binding, structural constituent of ribosome, nucleic acid metabolism, et al., which is crucial for maintaining the normal state of the cell metabolism process. RNA binding, structural constituent of ribosome, and nucleic acid metabolism were involved in the conventional DNA replication and RNA transcription process, which is extremely activated in tumor, like breast cancer, colon cancer, and glioma. Several potential targeted proteins of EEF1D, such as NFIC and RBMX, were reported to participate in the regulation of proliferation and EMT transition [25, 34, 35]. RBMX, also known as hnRNP G, was involved in various processes, like pre-mRNA splicing and posttranscriptional regulatory mechanism [36, 37], which were related to several vital processes of tumor initiation, progression, and metastasis. Alteration of the processes induced by aberrant expression of EEF1D may impair the indispensable nucleic acid metabolism and translational deregulation and finally impede tumor cell survival and progression including glioma. As for the biological process, multifarious cancer-related processes, such as nucleic acid metabolism, cell growth, protein metabolism, and mitosis, were the most common among the enriched pathways of the interacting proteins, consistent to the enriched results of the molecular function.

\section{Conclusion}

Overall, our results contribute to the knowledge of EEF1D effects on glioma and provide insights into the mechanism of glioma through mediating EMT and PI3K/Akt pathways. Therefore, blocking EEF1D could restrain EMT and PI3K/ Akt activity resulting in the suppression of cell growth and tumor progression. However, the detailed mechanism of EEF1D on glioma progression phenotype remains elusive and needs further investigation. Overall, our data provide evidence that EEF1D might serve as a potential therapeutic target for glioma.

\section{Data Availability}

The genomic expression data and clinicopathological data of 163 GBM tissues, 518 LGG tissues, and 207 normal brain tissues were downloaded from The Cancer Genome Atlas (TCGA, https://tcga-data.nci.nih.gov/TCGA). The putative EEF1D-interacting proteins were subjected to functional annotation analysis using FunRich analysis tool (Pathan et al. 2015).

\section{Conflicts of Interest}

All the authors declare that there was no conflict of interest.

\section{Authors' Contributions}

YS conceived and designed the experiments; CX and MZ performed the experiments; JL, ZW, and SD performed the analysis; JL, ZZ, and YC contributed to reference collecting; SX contributed to specimen collecting; CX contributed to writing. All authors have read and approved the final manuscript. Cheng Xie, Mingfeng Zhou, and Jie Lin have equally contributed to this work.

\section{Acknowledgments}

This study was supported by the National Nature Science Fund of China (Grant No. 81872064), Natural Science Foundation of Guangdong Province of China (Grant No. 2020A151501122), Science and Technology Program of Guangzhou, China (Grant No. 201903010048), and Outstanding Youths Development Scheme of Nanfang Hospital, Southern Medical University (Grant No. 2016J008).

\section{References}

[1] F. P. Barthel, P. Wesseling, and R. G. W. Verhaak, "Reconstructing the molecular life history of gliomas," Acta Neuropathologica, vol. 135, no. 5, pp. 649-670, 2018.

[2] J. Buckner, C. Giannini, J. Eckel-Passow et al., "Management of diffuse low-grade gliomas in adults - use of molecular diagnostics," Nature Reviews. Neurology, vol. 13, no. 6, pp. 340351, 2017.

[3] P. Diamandis and K. D. Aldape, "Insights from molecular profiling of adult glioma," Journal of Clinical Oncology, vol. 35, no. 21, pp. 2386-2393, 2017.

[4] M. Lim, Y. Xia, C. Bettegowda, and M. Weller, "Current state of immunotherapy for glioblastoma," Nature Reviews Clinical Oncology, vol. 15, no. 7, pp. 422-442, 2018.

[5] T. E. Dever and R. Green, "The elongation, termination, and recycling phases of translation in eukaryotes," Cold Spring Harbor Perspectives in Biology, vol. 4, no. 7, p. a013706, 2012.

[6] I. L. Flores, R. Kawahara, M. C. C. Miguel et al., "EEF1D modulates proliferation and epithelial-mesenchymal transition in oral squamous cell carcinoma," Clinical Science, vol. 130, no. 10, pp. 785-799, 2016. 
[7] M. Shuda, N. Kondoh, K. Tanaka et al., "Enhanced expression of translation factor mRNAs in hepatocellular carcinoma," Anticancer Research, vol. 20, no. 4, pp. 2489-2494, 2000.

[8] J. Piltti, J. Häyrinen, H. M. Karjalainen, and M. J. Lammi, "Proteomics of chondrocytes with special reference to phosphorylation changes of proteins in stretched human chondrosarcoma cells," Biorheology, vol. 45, no. 3-4, pp. 323-335, 2008.

[9] K. Ogawa, T. Utsunomiya, K. Mimori et al., "Clinical significance of elongation factor-1 delta mRNA expression in oesophageal carcinoma," British Journal of Cancer, vol. 91, no. 2, pp. 282-286, 2004.

[10] Y. Liu, Q. Chen, and J. T. Zhang, “Tumor suppressor gene 143-3sigma is down-regulated whereas the proto-oncogene translation elongation factor 1delta is up-regulated in nonsmall cell lung cancers as identified by proteomic profiling," Journal of Proteome Research, vol. 3, no. 4, pp. 728-735, 2004.

[11] M. De Bortoli, R. C. Castellino, X.-Y. Lu et al., "Medulloblastoma outcome is adversely associated with overexpression of EEF1D, RPL30, and RPS20 on the long arm of chromosome 8," BMC Cancer, vol. 6, no. 1, p. 223, 2006.

[12] J. Li, T. Cai, K. Deng, X. Wang, T. Sellis, and F. Xia, "Community-diversified influence maximization in social networks," Information Systems, vol. 92, p. 101522, 2020.

[13] T. A. O. T. A. O. CAI, J. Li, A. S. Mian, R. li, T. Sellis, and J. X. $\mathrm{Yu}$, "Target-aware Holistic Influence Maximization in Spatial Social Networks," in IEEE Transactions on Knowledge and Data Engineering, p. 1, 2020.

[14] Y. Song, S. Zheng, J. Wang et al., "Hypoxia-induced PLOD2 promotes proliferation, migration and invasion via PI3K/Akt signaling in glioma," Oncotarget, vol. 8, no. 26, pp. 4194741962, 2017.

[15] H. Zhang, S. P. Young, C. Auray-Blais, P. J. Orchard, J. Tolar, and D. S. Millington, "Analysis of glycosaminoglycans in cerebrospinal fluid from patients with mucopolysaccharidoses by isotope-dilution ultra-performance liquid chromatographytandem mass spectrometry," Clinical Chemistry, vol. 57, no. 7, pp. 1005-1012, 2011.

[16] D. S. M. Chatzileontiadou, M. Samiotaki, A. N. Alexopoulou et al., "Proteomic analysis of human angiogenin interactions reveals cytoplasmic PCNA as a putative binding partner," Journal of Proteome Research, vol. 16, no. 10, pp. 3606-3622, 2017.

[17] J. M. Gao, L. Z. Huang, Z. G. Huang, and R. Q. He, “Clinical value and potential pathways of miR-183-5p in bladder cancer: a study based on miRNA-seq data and bioinformatics analysis," Oncology Letters, vol. 15, no. 4, pp. 5056-5070, 2018.

[18] H. Yi, X. Zheng, J. Song, R. Shen, Y. Su, and D. Lin, "Exosomes mediated pentose phosphate pathway in ovarian cancer metastasis: a proteomics analysis," International Journal of Clinical and Experimental Pathology, vol. 8, no. 12, pp. 15719-15728, 2015.

[19] H. Shen, J. Huang, H. Pei et al., "Comparative proteomic study for profiling differentially expressed proteins between Chinese left- and right-sided colon cancers," Cancer Science, vol. 104, no. 1, pp. 135-141, 2013.

[20] J. Keenan, L. Murphy, M. Henry, P. Meleady, and M. Clynes, "Proteomic analysis of multidrug-resistance mechanisms in adriamycin-resistant variants of DLKP, a squamous lung cancer cell line," Proteomics, vol. 9, no. 6, pp. 1556-1566, 2009.

[21] L. Gyenis, J. S. Duncan, J. P. Turowec, M. Bretner, and D. W. Litchfield, "Unbiased functional proteomics strategy for pro- tein kinase inhibitor validation and identification of bona fide protein kinase substrates: application to identification of EEF1D as a substrate for CK2," Journal of Proteome Research, vol. 10, no. 11, pp. 4887-4901, 2011.

[22] G. Sivan, R. Aviner, and O. Elroy-Stein, "Mitotic modulation of translation elongation factor 1 leads to hindered tRNA delivery to ribosomes," The Journal of Biological Chemistry, vol. 286, no. 32, pp. 27927-27935, 2011.

[23] D. D. Cheng, S. J. Li, B. Zhu, S. M. Zhou, and Q. C. Yang, "EEF1D overexpression promotes osteosarcoma cell proliferation by facilitating Akt-mTOR and Akt-bad signaling," Journal of Experimental \& Clinical Cancer Research, vol. 37, no. 1, p. 50, 2018.

[24] X. Liu, J. Yang, Q. Zhang, and L. Jiang, "Regulation of DNA methylation on EEF1D and RPL8 expression in cattle," Genetica, vol. 145, no. 4-5, pp. 387-395, 2017.

[25] X. Huang, X. Xu, Bringas P Jr, Y. P. Hung, and Y. Chai, “Smad4Shh-Nfic signaling cascade-mediated epithelial-mesenchymal interaction is crucial in regulating tooth root development," Journal of Bone and Mineral Research, vol. 25, no. 5, pp. 1167-1178, 2010.

[26] I. C. Iser, M. B. Pereira, G. Lenz, and M. R. Wink, "The epithelial-to-mesenchymal transition-like process in glioblastoma: an updated systematic review and in silico investigation," Medicinal Research Reviews, vol. 37, no. 2, pp. 271313, 2017.

[27] U. D. Kahlert, G. Nikkhah, and J. Maciaczyk, "Epithelial-tomesenchymal(-like) transition as a relevant molecular event in malignant gliomas," Cancer Letters, vol. 331, no. 2, pp. 131-138, 2013.

[28] M. H. Meel, S. A. Schaper, G. J. L. Kaspers, and E. Hulleman, "Signaling pathways and mesenchymal transition in pediatric high-grade glioma," Cellular and Molecular Life Sciences, vol. 75, no. 5, pp. 871-887, 2018.

[29] B. S. Huang, Q. Z. Luo, Y. Han, D. Huang, Q. P. Tang, and L. X. $\mathrm{Wu}$, "MiR-223/PAX6 axis regulates glioblastoma stem cell proliferation and the chemo resistance to TMZ via regulating PI3K/Akt pathway," Journal of Cellular Biochemistry, vol. 118, no. 10, pp. 3452-3461, 2017.

[30] A. Rodríguez-García, P. Samsó, P. Fontova et al., "TGF- $\beta 1$ targets Smad, p38 MAPK, and PI3K/Akt signaling pathways to induce PFKFB3 gene expression and glycolysis in glioblastoma cells," The FEBS Journal, vol. 284, no. 20, pp. $3437-$ 3454, 2017.

[31] X. Li, C. Wu, N. Chen et al., "PI3K/Akt/mTOR signaling pathway and targeted therapy for glioblastoma," Oncotarget, vol. 7, no. 22, pp. 33440-33450, 2016.

[32] E. Majewska and M. Szeliga, "AKT/GSK3 $\beta$ signaling in glioblastoma," Neurochemical Research, vol. 42, no. 3, pp. 918-924, 2017.

[33] S. Venkatesan, M. L. M. Lamfers, C. M. F. Dirven, and S. Leenstra, "Genetic biomarkers of drug response for smallmolecule therapeutics targeting the RTK/Ras/PI3K, p53 or $\mathrm{Rb}$ pathway in glioblastoma," CNS oncology, vol. 5, no. 2, pp. 77-90, 2016.

[34] M. Fane, L. Harris, A. G. Smith, and M. Piper, "Nuclear factor one transcription factors as epigenetic regulators in cancer," International Journal of Cancer, vol. 140, no. 12, i, pp. 26342641, 2017.

[35] J. Nilsson, K. Helou, A. Kovács et al., "Nuclear Janus-activated kinase 2/nuclear factor 1-C2 suppresses tumorigenesis and 
epithelial-to-mesenchymal transition by repressing Forkhead box F1," Cancer Research, vol. 70, no. 5, pp. 2020-2029, 2010.

[36] F. Martínez-Arribas, D. Agudo, M. Pollán et al., "Positive correlation between the expression of X-chromosomeRBM genes (RBMX, RBM3, RBM10) and the proapoptoticBax gene in human breast cancer," Journal of Cellular Biochemistry, vol. 97, no. 6, pp. 1275-1282, 2006.

[37] K. H. Shin, R. H. Kim, R. H. Kim, M. K. Kang, and N. H. Park, "hnRNP G elicits tumor-suppressive activity in part by upregulating the expression of Txnip," Biochemical and Biophysical Research Communications, vol. 372, no. 4, pp. 880-885, 2008. 